\author{
Bilal Ahmed* \\ FCPS, FFD RCSI-II, BDS, PhD Scholar, Associate \\ Professor Prosthodontics, Army Medical College, \\ National University of Science \& Technology (NUST) \\ Islamabad, Pakistan
}

Dates: Received: 23 August, 2014; Accepted: 10 September, 2014; Published: 12 September, 2014

*Corresponding author: Bilal Ahmed, House No. 47 Block No. 30, Sargodha (40100), Pakistan, E-mail: drbilalahmed79@hotmail.com; drbilalahmed79@ amcollege.nust.edu.pk

www.peertechz.com

ISSN: 2455-5282

Keywords: Maxillofacial defects; Prosthetic rehabilitation; Acrylic nasal rehabilitation; Basal cell carcinoma

\author{
Case Report
}

\section{Saving Faces Changing Lives the Art of Maxillofacial Prosthodontics Case Series Comparing Two Different Materials}

\section{Introduction}

Defects of maxillofacial region can result from trauma, surgery, malignancies, congenital and acquired anomalies, infections and burns. The rehabilitation of these defects can be achieved either surgically, prosthetically or through a combination of both [1].

Restoration of large facial defects is a challenge both for the maxillofacial plastic surgeons and maxillofacial prosthodontists. Prosthetic restoration of facial defect is a treatment of choice where surgical reconstruction is not possible. Pre-surgical records like photographs, mounted extra-oral casts and facial cast if available, could facilitate the fabrication of the prosthesis [2].

Prosthodontic results are limited by the materials used in the construction and fabrication of facial prostheses as success depends mainly on the physical and mechanical properties of the material used [3]. Materials commonly used for fabrication of facial prostheses are acrylic resins, acrylic copolymers, vinyl polymers, polyurethane elastomers and silicone elastomers, but none of them fulfill all the requirements for a satisfactory prosthesis [4]

Acrylic resin has advantages of color stability, remains serviceable for upto 2 years and can be relined if required. However, silicones remain the more widely used materials for facial restorations because of their good surface texture and hardness [5]. More recently, the use of tulle has shown improved edge strength in facial prostheses [6].

Recently CAD/CAM (computer aided design and computer aided manufacturing) system for fabrication of oral and maxillofacial prostheses is being used. However, its use is limited due to its complexity, cost and non-availability at many centres [7].
Retention in facial prosthesis can be achieved by biocompatible adhesives, mechanically by engaging anatomical undercuts, attaching the prosthesis to the patient's eyeglasses, use of straps and head bands, magnets or Osseo integrated implant retained titanium screws. Adhesives are the most commonly used materials for retention but the weight of larger prostheses may prohibit or limit their use [8]

These reports present cases of prosthetic rehabilitation of the lost nasal component of the face secondary to nasal malignant lesion using patient's eyeglasses for biomechanical retention and secondary to nasal leishmaniasis using the maxillofacial prosthesis.

\section{Case 1}

A 63 years old male daily wager patient was referred to the department of prosthodontics for rehabilitation of his lost nasal portion of the face. The patient presented with history of surgical resection of the nose three months earlier due to an aggressive Basal Cell carcinoma. The patient was operated by the plastic surgery department later on using a forehead flap for nasal reconstruction, but this attempt got failed (Figure 1).

After detailed history, thorough examination and discussion with the patient, it was planned to fabricate a nasal prosthesis in acrylic resin material using a donor impression technique [1]. Initial impressions were recorded in alginate impression materials using facial moulage technique and a cast was fabricated. An alginate impression of the nasal region was recorded from one of the relatives of the patient and a wax try in was performed. After adjustments of the fitting details, a prosthesis was fabricated in dentine colored acrylic resin using conventional flasking and curing technique. The prosthesis was again tried in the patient's nasal cavity and then fixed with his eye glasses using the same color cold cure acrylic resin (Figure 3). Then 
the final shade matching and tinting was done using acrylic color kit (Maries acrylic color, Shanghai China) (Figures 1,2). The patient was instructed about the maintenance procedures and a regular follow up was scheduled. Our patient was comfortable and satisfied with the appliance. He was able to cope with his daily social interactions and was feeling more confident and comfortable.

\section{Case 2}

This patient was referred to the outpatient department of de 'Montmorency College of Dentistry, Lahore from the plastic surgery department of Mayo Hospital Lahore [1] (Figure 4).

The patient presented a ten year long history of lost external nose as well as a perforation in the hard palate making a large oro-nasal communication. There was a history of repeated infections in the mid facial region, extending into the nasal septum and floor of the nasal cavity, which ultimately led to total ablation of the external nasal component and formed a defect in the palate as well. No medical

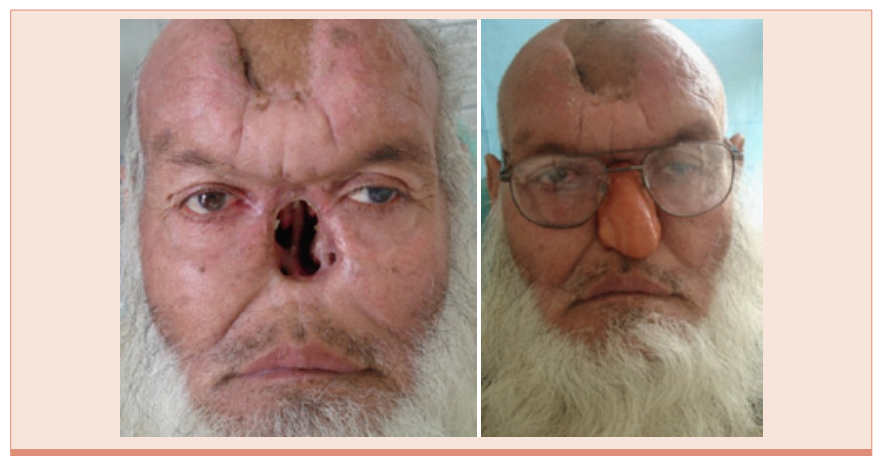

Figure 1: Pre-operative and Post-operative Frontal views.

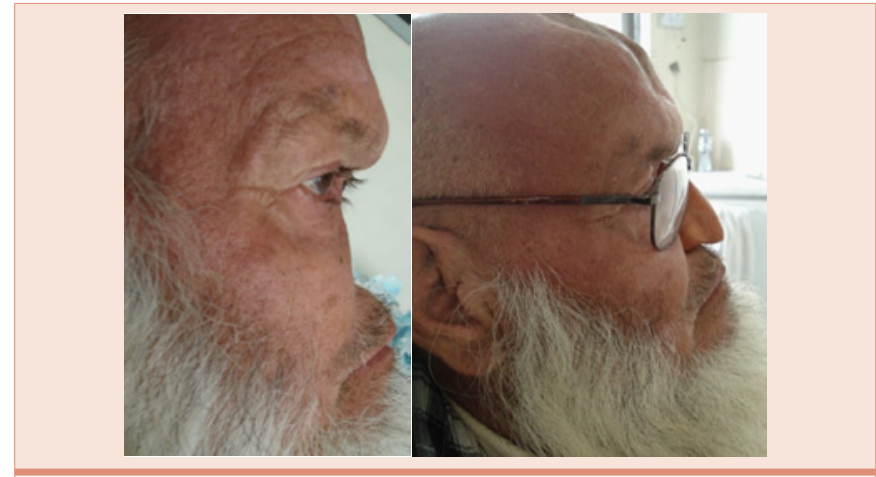

Figure 2: Pre-operative and Post-operative Lateral views.

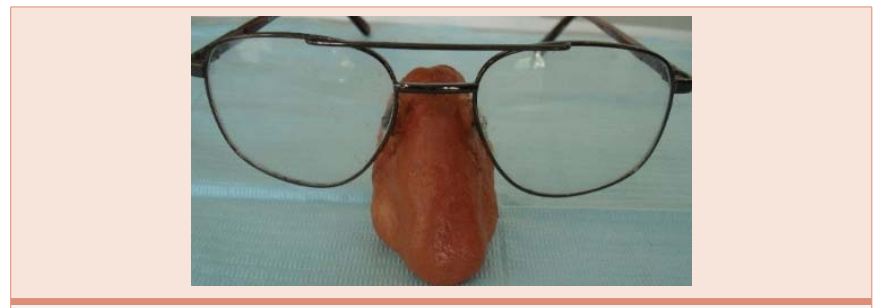

Figure 3: Nasal prosthesis with eye glasses

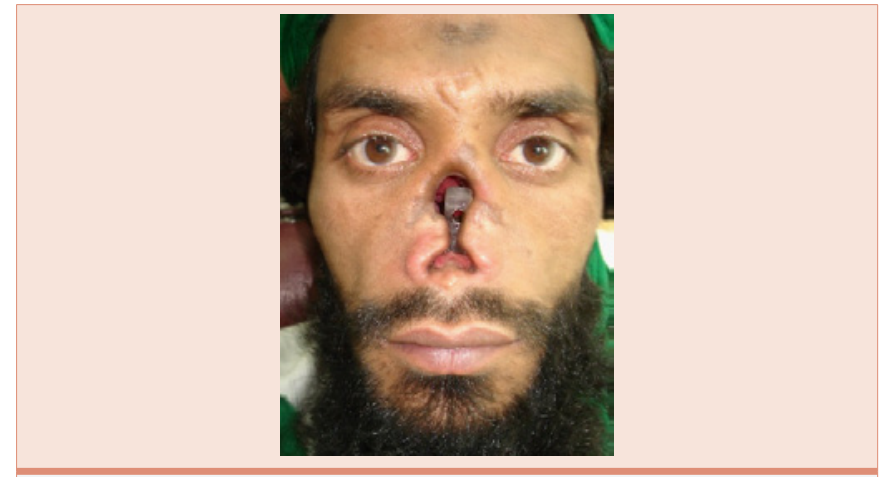

Figure 4: Pre-operative view with palatal obturator in place.

record was available with the patient. However, these problems are usually characteristic features of uncontrolled muco-cutaneous nasal leishmaniasis. This patient presented a serious functional as well as aesthetic dilemma. He used to cover his face with a piece of cloth all the time to hide the big nasal defect. His phonetics were severely compromised and his speech was totally incomprehensible. He was unable to eat and drink properly because of nasal regurgitation. On extra oral examination, there was no sign of any ulceration. External nasal component including septum and bridge of the nose was totally lost. On intraoral examination, there was an ovoid perforation about $1 \mathrm{~cm}$ wide in the midline of the hard palate.

After discussion with the patient, a two part prosthesis was planned. Initial intra oral impression was recorded using alginate impression material. A removable acrylic palatal plate with a nasal extension was constructed and retained using Adam's clasp assemblies on the maxillary first molar bilaterally. A hole was created in the nasal extension and a magnetic sleeve was placed (Figures 5, 6).

A metallic component was placed and a facial impression was recorded using pickup impression technique in alginate. Boxing of the impression was done on the face of the patient [1].

Impression was poured in extra hard plaster. After setting of the plaster model, undercuts were blocked out and the nasal prosthesis was fabricated in modeling wax. Try-in was done and final prosthesis was fabricated in a medical grade silicone material (MOLLOMED). After finishing and polishing, final shade matching and tinting was done on chair side. The prosthesis was very stable and retentive because of magnetic retainers as well as undercut extensions of the silicone. However, to mask the glare and margins of the prosthesis, eyeglasses were offered to the patient which further added to the retention. Patient was also advised to use the silicone adhesives to seal the margins which will prevent the leakage of air and secretions from the nasal defect as well as aid in retention [1].

\section{Discussion}

Plastic and reconstructive surgery is definitely a treatment of choice for patients with facial defects, but for larger defects with extensive anatomical loss, a more suitable alternative is prosthetic rehabilitation.

This article described the process for fabrication and construction of large nasal prosthesis, by the use of simple available materials. This 


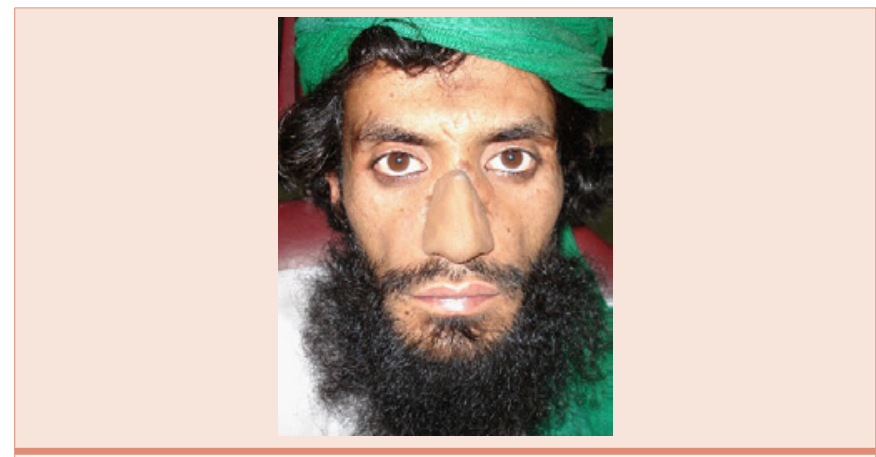

Figure 5: Post-operative view.

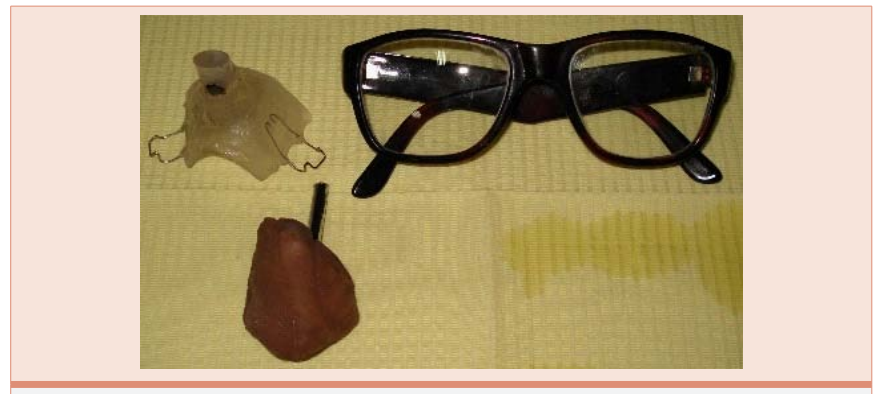

Figure 6: Nasal prosthesis, Palatal prosthesis and Glasses.

was fabricated and delivered to the patients within a week to achieve the objectives outlined.

In this large and extensive defect, which did not have much tissue support, the facial prosthesis made in acrylic resin gave satisfactory results because of its reduced weight, convenience of cleaning and developed shade and texture.

In these case, the final results after prosthetic treatment were reasonable both from a cosmetic point as well as from patient's functional efficiency. However, the difficulty in maxillofacial rehabilitation of large facial defects often involves the compromise of cosmetics versus functional adequacy and therefore, the patient can be the only one to determine which aspect of the prosthesis contributes to his/her quality of life [9].

The objective in treating such patients is to restore the lost natural tissue immediately or later after surgery or nasal ablation so as to maintain appearance, morale and confidence of the patient and to facilitate social acceptance among the public and their families, which these patients from maxillofacial malignancies lack.

It is advocated that lack of highly sophisticated equipment and materials should not be a limiting factor in the rehabilitation of the sufferings of these fellow human beings. Even, simple available materials like acrylic resins, silicones and polyether can be extremely helpful in the fabrication of maxillofacial prostheses if facilities like CAD-CAM or maxillo-facial implants are not accessible. These simple removable options can be extremely cost effective, conservative and without aggressive side effects, so they are enthusiastically accepted by the patients and their relatives. However, a thorough patient evaluation is always mandatory, to determine the quality of restorations required as many times these simple, removable options may also jeopardize the socioeconomic and personal comfort of the patient. In these cases, referral to more advanced and sophisticated treatment modalities should always be considered.

\section{References}

1. Ahmed B, Mahmood A, Hussain M, Amin M, Yazdanie N (2010) Rehabilitation of nose using silicone based maxillofacial prosthesis. J Coll Physician Surg Pak 20: 65-67.

2. Futran ND, Mendez E (2006) Developments in reconstruction of midface and maxilla. Lancet Oncol 7: 249-258.

3. Bou C, Pomar P, Miquel JL, Poisson P (2006) [Maxillo-facialprostheses: an issue in public health]. Odontostomatol Trop 29: 34-40.

4. French, Goiato MC, Pesqueira AA, dos Santos DM, Antenucci RM, et al. (2008) Evaluation of dimensional change and detail reproduction in silicones for facial prostheses. Acta Odontol Latinoam 21: 85-88.

5. Mortellaro C, Garagiola U, Lucchina AG, Grivetto F, Milone G, et al. (2006) The use of silicon elastomer in maxillofacial rehabilitation as a substitute for or in conjunction with resins. J Craniofac Surg 17: 152-162.

6. Karayazgan B, Gunay Y, Evlioglu G (2003) Improved edge strength ina facial prosthesis by incorporation of tulle: a clinical report. J Prosthet Dent 90: 526529.

7. Tsuji M, Noguchi N, Ihara K, Yamashita Y, Shikimori M, et al. (2004) Fabrication of a maxillofacial prosthesis using a computer-aided design and manufacturing system. J Prosthodontics 13: 179-183.

8. Ciocca L, Maremonti P, Bianchi B, Scotti R (2007) Maxillofacial rehabilitation after rhinectomy using two different treatment options: clinical reports. J Oral Rehabil 34: 311-315.

9. Ahmed B, Butt AM, Yazdanie N (2014) DENTAL HEALTH \& QUALITY OF LIFE. ISBN No. 978-3-659-54719-5. Lambert Academic Publishing Germany 2014: 77-78. 